\title{
Swift observations of black hole candidate, XTE J1752-223, during outburst
}

\author{
Peter A. Curran* ${ }^{\dagger}$ \\ AIM, CEA/DSM - CNRS, Irfu/SAP, Centre de Saclay, Bat. 709, FR-91191 Gif-sur-Yvette Cedex, \\ France \\ E-mail: peter.curran@cea.fr
}

\section{T.J. Maccarone}

School of Physics and Astronomy, University of Southampton, Southampton, Hampshire, SO17 1BJ, UK

\section{P. Casella}

School of Physics and Astronomy, University of Southampton, Southampton, Hampshire, SO17 1BJ, UK

\begin{abstract}
Here we summarise the Swift broadband observations of the recently discovered X-ray transient and black hole candidate, XTEJ1752-223, obtained over the period of outburst from October 2009 to June 2010. We offer a phenomenological treatment of the spectra as an indication of the canonical spectral state of the source during different periods of the outburst. We find that the high energy hardness-intensity diagrams over two separate bands follows the canonical behavior, confirming the spectral states. From Swift-UVOT data we confirm the presence of an optical counterpart which displays variability correlated, in the soft state, to the X-ray emission observed by Swift-XRT. The optical counterpart also displays hysteretical behaviour between the states not normally observed in the optical bands, suggesting a possible contribution from a synchrotron emitting jet to the optical emission in the rising hard state. Our XRT timing analysis shows that in the hard state there is significant variability below $10 \mathrm{~Hz}$ which is more pronounced at low energies, while during the soft state the level of variability is consistent with being minimal.These properties of XTEJ1752-223 support its candidacy as a black hole in the Galactic centre region.
\end{abstract}

8th INTEGRAL Workshop "The Restless Gamma-ray Universe"

September 27-30 2010

Dublin Castle, Dublin, Ireland

\footnotetext{
* Speaker.

${ }^{\dagger}$ Mullard Space Science Laboratory, University College of London, Holmbury St Mary, Dorking, Surrey RH5 6NT, UK
} 

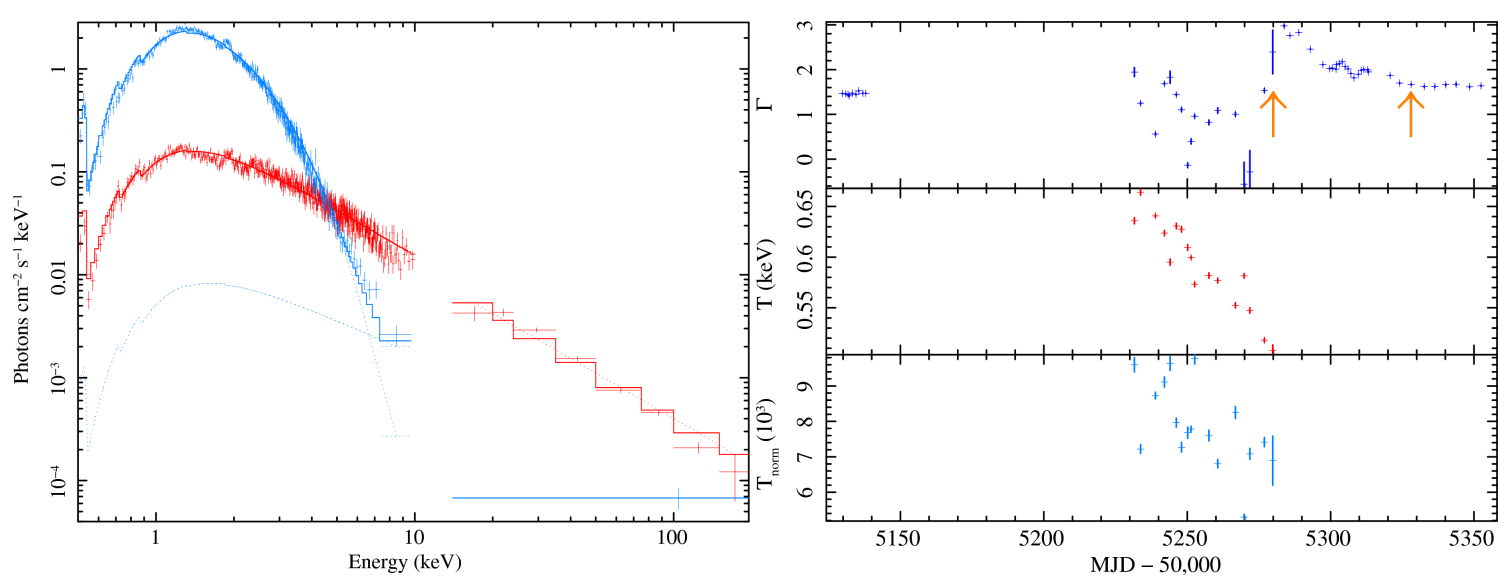

Figure 1: Left: Example XRT-BAT spectra in the power-law dominated, hard state (red; MJD 55131) and in the soft state (blue; MJD 55248) which displays a strong thermal component. Right: Best fit parameters of the joint XRT-BAT spectral fits: power law photon index, $\Gamma$, thermal component temperature, $T$, and thermal component normalization, $T_{\text {norm }}$, in units of $10^{3}$. Note that the photon indices, in the period where there is a thermal component, are not reliable. The absence of data from MJD 55139 to 55231 is due to the various instruments becoming sun-constrained. The arrows signify the dates between which the source is transitioning from the soft state to the late, hard state.

\section{XTE J1752-223: Introduction}

A new X-ray transient and black hole candidate in the Galactic center region, XTEJ1752223 was detected on 2009-10-23 at 19:55 UT (MJD 55128.33) by RXTE[7]. The high energy, variable emission of the source was confirmed in the following days by Swift-XRT \& BAT[6] and RXTE[10, 11] as well as by MAXI/GSC[9] and Fermi/GBM[14]. An optical and nIR counterpart was proposed $[12,13]$ and a radio source coincident with the X-ray position, later confirmed as the jet [15], was detected[1]. The source was observed to have undergone a state transition in mid-January 2010 (MJD 55210), from a spectrally hard to a spectrally soft X-ray state[3]. This was identified by a decoupling of high energy ( $\gtrsim 4 \mathrm{keV})$ and low energy $(\lesssim 4 \mathrm{keV})$ MAXI light curves which had previously traced each other, and by the dominance of a thermal component in spectra. The source was observed to have reverted to a hard state at the end of March 2010 (MJD $\sim 55280$ ) [8], after the low energy light curve decreased to trace once more the high energy light curve; the thermal component was no longer dominant. Here we summarise our paper[2] detailing the Swift - Burst Alert Telescope (BAT), X-ray Telescope (XRT) and Ultraviolet/Optical Telescope (UVOT) - monitoring observations of XTE J1752-223, obtained over the period of the outburst. Based on these data, we identify the periods of the various states[5] and compare the behavior of the major photometric, spectral and timing properties during these states to those expected from black hole X-ray binaries.

\section{Defining the states}

The states are defined by the XRT-BAT spectral analysis (Figure 1) which shows that in the initial hard state (MJD 55131 - 55138), the spectra are dominated by a single, hard power law 

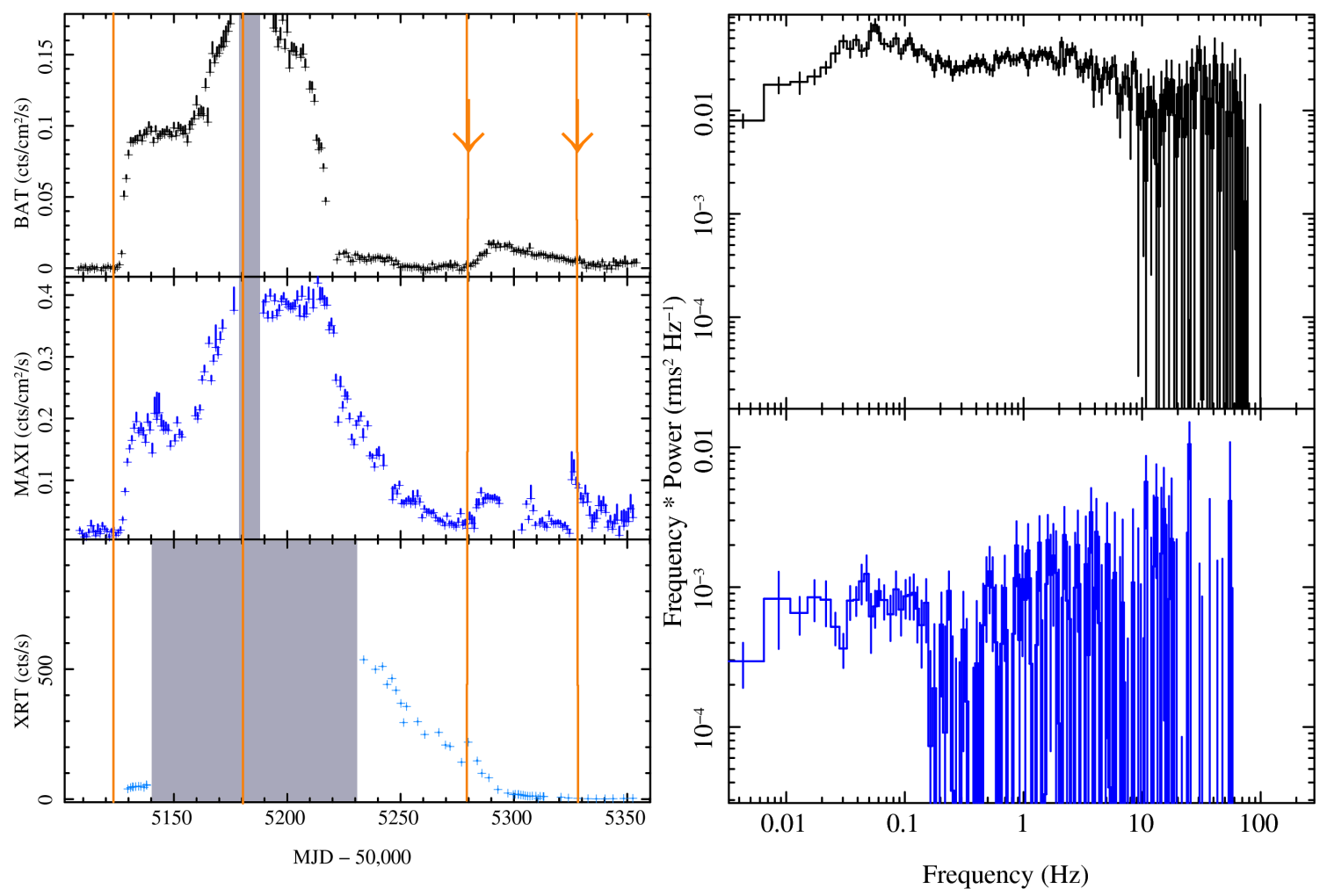

Figure 2: Left: High energy (Swift-BAT [15-150 keV], MAXI [4-10 keV], Swift-XRT [0.3-10 keV]) light curves during the period of outburst. The absence of data around MJD 55180 (shaded area) is due to the various instruments becoming sun-constrained. The orange lines signify the dates of the state transitions: quiescence - hard state - soft state - intermediate state - hard state. Right: The average power density spectrum (PDS) for XRT light curve in the initial hard state (upper; RMS 54\%) exhibits the aperiodic variability of the light curve not present in the average soft state PDS (lower; RMS $<12 \%$ ).

component of photon index, $\Gamma=1.46$. In the soft state (MJD 55233 - 55280) there is a significant additional contribution from a thermal component at $\sim 0.6 \mathrm{keV}$. In the intermediate state (MJD 55283 - 55328), a thermal component in no longer supported though the power law is still decreasing until it reaches its final, hard state (MJD 55329 - 55352) value of $\Gamma=1.64$ where the column density is fixed to that of the initial hard state. This steeper value at late times, like the HR value (section 5), shows that the source has not returned to it's initial state or that the late hard state has different spectral properties, such as column density, than the initial hard state.

In the high energy Swift-BAT [15-150 keV], MAXI [4-10keV] and Swift-XRT [0.3-10 keV] light curves (Figure 2) during the period of outburst, the absence of data at MJD 55139-55233 (shaded area) is due to the various instruments becoming sun-constrained. The orange lines signify the dates of the state transitions: quiescence - hard state - soft state - intermediate state - hard state. It is clear from these that there is an excess of soft/low energy photons in the soft state as exhibited by the decoupling of high energy and low energy light curves which had previously traced each other. Unfortunately, due to the sun constraint, the intermediate state between the initial hard state and the soft state was unobserved. 

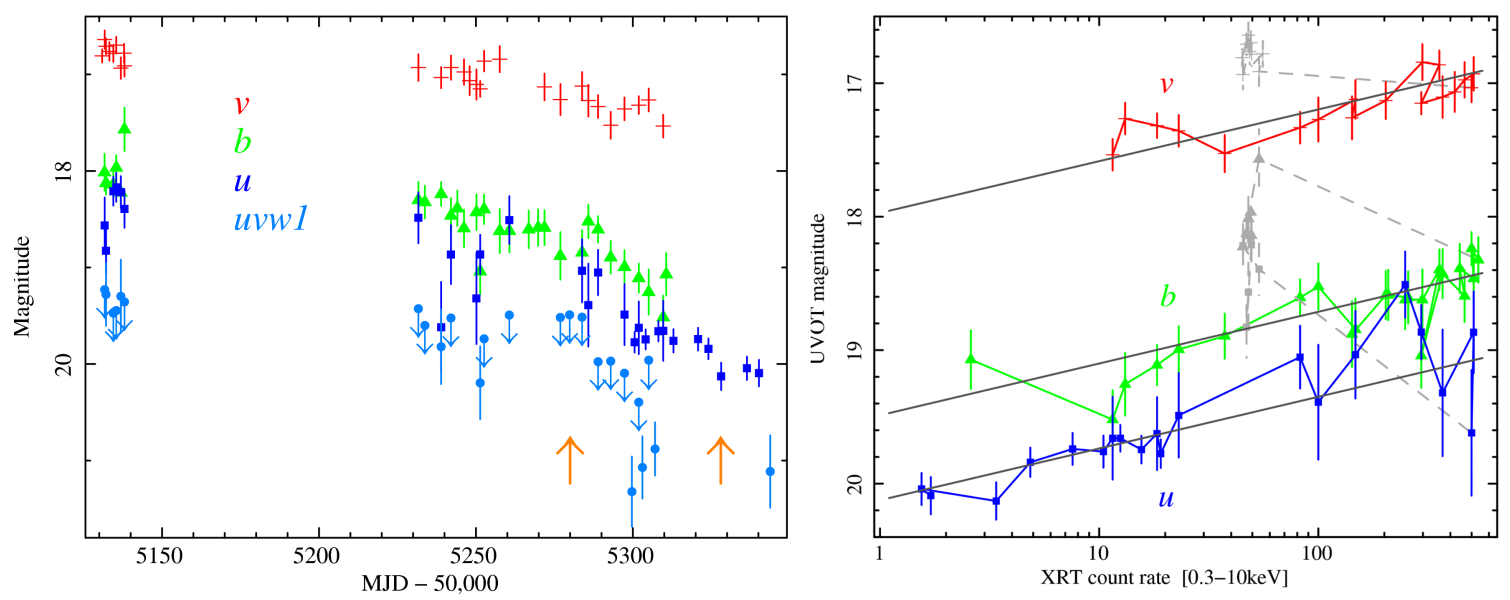

Figure 3: Left: UVOT $v$ (crosses), $b$ (triangles), $u$ (squares) and $u w 1$ (circles with $3 \sigma$ limits) band light curves for the source show variability; dimming by 1 magnitude or more from the start to the end of observations. The up-pointing arrows signify the dates between which the source is transitioning from the soft state to the late, hard state. Right: UVOT magnitude in three filters versus XRT count rate. Data points in grey indicate observations before MJD 55230, i.e., the initial hard state. The solid black lines represent the simultaneous power law fit to the data after MJD 55230.

\section{Optical counterpart \& hysteresis}

UVOT $v, b, u$ and $u v w 1$ band light curves (Figure 3) for the proposed optical counterpart allowed us to confirm the association with XTE J1752-223 due to their variability. The accurate position of the counterpart was derived from a deep (1853 s) $v$ band image as 17:52:15.08 -22:20:32.9 (J2000; 0.31 arcsecond $1 \sigma$ error). Given the unknown Galactic extinction to the source and the quality of the data, we cannot examine the true colours or spectral shape of the optical source, neither can we confirm any possible spectral changes over the outburst. Assuming a power law spectrum, the spectral index could be anywhere between a rising value of 2.0 and a decreasing value of 5.3.

The correlation (solid black lines) between the UVOT magnitudes in three bands and the XRT count rate during the soft state further confirm the association between the proposed counterpart and XTEJ1752-223. Data points in grey indicate observations during the initial hard state and clearly exhibit a hysteresis with the later soft state data at a similar count rate. This indicates an additional emission component from, e.g., a jet which would be quenched in the soft state and weak at low X-ray luminosities.

\section{X-ray variability}

The average power density spectrum (PDS; Figure 2) for XRT light curve in the hard state exhibits an aperiodic variability of the light curve (RMS 54\%) at frequencies up to $10 \mathrm{~Hz}$, though no QPOs are detected. The average soft state PDS exhibits only minimum variability and only at frequencies below $0.2 \mathrm{~Hz}$ (RMS $<12 \%$ ). These PDS help confirm the identified states as they exhibit the same properties as observed in other black hole X-ray binaries. We also find that, in the initial hard state, there is an excess of variability at low energies: the low energy (0.3-1.5 keV) light 

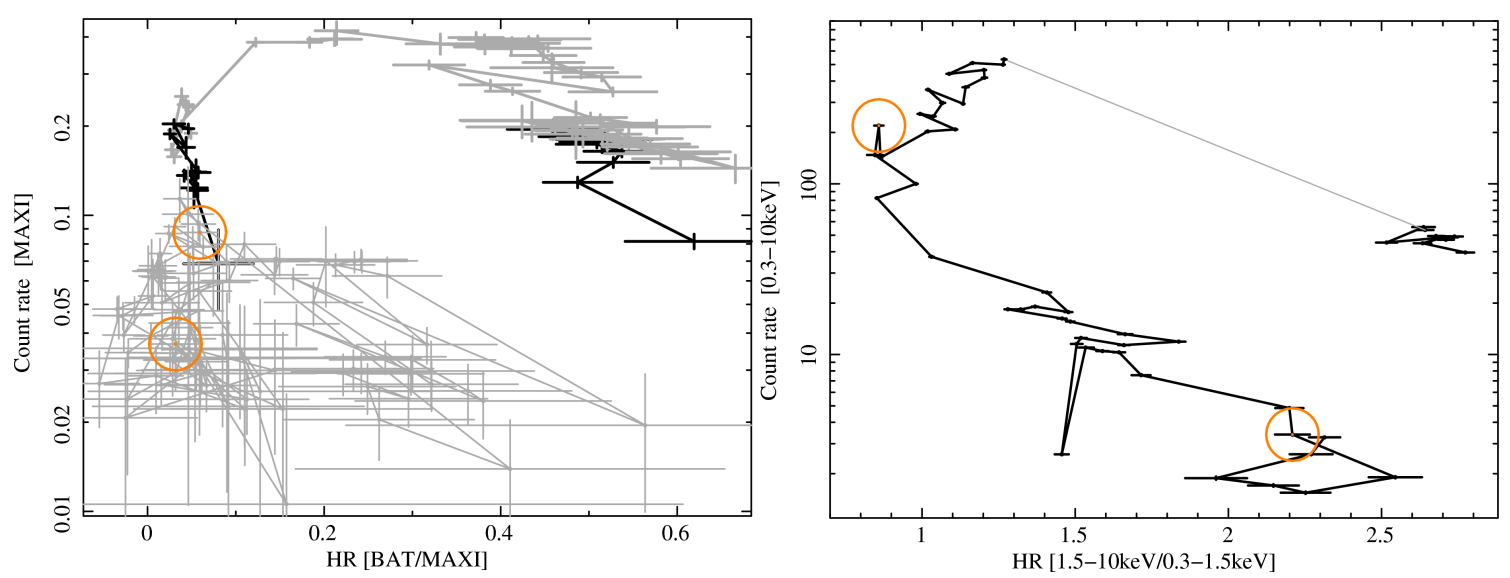

Figure 4: Hardness-Intensity diagrams (HID) for both BAT/MAXI (left) and XRT (right). The grey lines, at high count rates in both plots show the time during which the XRT was unable to observe (MJD 5513955233), while the circles show the start and end of the transition from the soft state. Note that at low count rates $(\lesssim 0.1)$ the BAT/MAXI HR is not reliable; the later, low count rate, data points are shaded for clarity of the plot.

curves having $20 \%$ higher RMS than the high energy (1.5-10 keV) light curves. This may suggest a contribution from intrinsic disk variability which is not obvious from the spectral fitting (section 2). More detailed spectral fitting and better constrained column density are required to test this possibility.

\section{Hardness-Intensity Diagrams}

In the Hardness-Intensity diagrams (HID; Figure 4) for both BAT/MAXI and XRT, the grey lines, at high count rates, show the time during which the XRT was unable to observe, while the circles show the start and end of the intermediate state between the soft and hard states. Note that at low count rates $(<0.1)$ the BAT/MAXI HR is not reliable; the later, low count rate, data points are shaded for clarity of the plot. Both HID are consistent with the canonical trajectory of black hole binaries[4]. It is clear from the XRT HID that the hardness has yet to increase to the original value, indicating that the source has not yet returned to its original hard state, though the count rate is lower than it was in that rising hard state, or that the late hard state has different spectral properties (e.g. column density).

\section{Conclusions}

Swift observations of the first, and so far only, observed outburst of XTE J1752-223 allow us to confirm and refine the epochs of the canonical X-ray states. The observations also allow us to confirm the optical counterpart for which we were able to produce a sub-arcsecond position. We show that there is a correlation between optical and X-ray emission in the soft state as well as a hysteresis effect where, for a given X-ray count rate, the magnitude in the rising hard state is significantly higher than that in the soft state. This is similar to the hysteretical behaviour observed in the nIR for a number of transients though it is not normally observed in the optical bands. The 
discussed X-ray and optical, photometric, spectral and timing properties of XTE J1752-223 support its candidacy as a black hole in the Galactic centre region.

\section{References}

[1] Brocksopp, C., Corbel, S., Tzioumis, T., \& Fender, R. 2009, ATel, 2278

[2] Curran, P. A., Maccarone, T. J., Casella, P., Evans, P. A, Landsman, W., Krimm, H. A., Brocksopp, C., \& Still, M. 2010, MNRAS, in press

[3] Homan, J. 2010, ATel, 2387, 1

[4] Homan, J., \& Belloni, T. 2005, Ap\&SS, 300, 107

[5] McClintock, J. E., \& Remillard, R. A. 2006, Black hole binaries, ed. Lewin, W. H. G. \& van der Klis, M. 157

[6] Markwardt, C. B., Barthelmy, S. D., Evans, P. A., \& Swank, J. H. 2009a, ATel, 2261

[7] Markwardt, C. B., et al. 2009b, ATel, 2258

[8] Muñoz-Darias, T., Motta, S., Belloni, T., \& Homan, J. 2010a, ATel, 2518, 1

[9] Nakahira, S., et al. 2009, ATel, 2259

[10] Remillard, R. A., \& The ASM Team at MIT. 2009, ATel, 2265

[11] Shaposhnikov, N., Markwardt, C. B., \& Swank, J. H. 2009, ATel, 2269, 1

[12] Torres, M. A. P., Jonker, P. G., Steeghs, D., Yan, H., Huang, J., \& Soderberg, A. M. 2009a, ATel, 2263

[13] Torres, M. A. P., Steeghs, D., Jonker, P. G., Thompson, I., \& Soderberg, A. M. 2009b, ATel, 2268

[14] Wilson-Hodge, C. A., Camero-Arranz, A., Case, G., Chaplin, V., \& Connaughton, V. 2009, ATel, 2280

[15] Yang, J., Brocksopp, C., Corbel, S., Paragi, Z., Tzioumis, T., \& Fender, R. P. 2010, MNRAS, in press 